\title{
Beyond-the-Standard-Model searches at HL-LHC
}

\section{Giuliano Gustavino*}

University of Oklahoma, Norman, USA

E-mail: giuliano.gustavinodcern.ch

The High-Luminosity Large Hadron Collider (HL-LHC) is expected to deliver the unprecedented integrated luminosity of $3 \mathrm{ab}^{-1}$ at the centre-of-mass energy of $14 \mathrm{TeV}$. This will provide a unique opportunity in the Beyond-the-Standard-Model (BSM) search program to explore still unknown physics scenarios. The full potential of the foreseen detector upgrades can be exploited by new specialised strategies to detect new physics phenomena. In this context an overview of some of the BSM searches planned by the ATLAS and CMS experiments is addressed, highlighting the sensitivity reaches and the possible improvements with respect to the current Run-2 results.

7th Annual Conference on Large Hadron Collider Physics - LHCP2019

20-25 May, 2019

Puebla, Mexico

\footnotetext{
*Speaker.
} 


\section{Introduction}

In the coming years the LHC and the ATLAS [1] and CMS [2] experiments will undergo significant upgrades. The foreseen integrated luminosity which will be collected through 2036 corresponds to $3 \mathrm{ab}^{-1}, 10$ times the integrated luminosity expected for the LHC Runs 1-3 combined. This unprecedented luminosity will be crucial for precision measurements in the Higgs sector and for increasing the discovery potential at the energy frontier. Among the most important detector upgrades foreseen for both the experiments are:

- a new trigger and data acquisition (TDAQ) system,

- a new inner tracker with an extended coverage of $|\eta|<4$,

- an improved muon system acceptance,

- a high granularity timing detector.

These upgrades will be crucial in order to maintain or improve the object identification and reconstruction performance in the challenging environment expected at a centre-of-mass energy of $14 \mathrm{TeV}$ with an instantaneous luminosity up to $5-7 \times 10^{34} \mathrm{~cm}^{-2} \mathrm{~s}^{-1}$ and an average number of $p p$ interactions (pile-up) of 140 to 200.

In this context, some of the most relevant new physics searches are discussed, highlighting the sensitivity increase due to several aspects, from the large dataset size to the potential of the new detector upgrades. The ATLAS and CMS collaborations compiled physics projections for BSM searches at HL-LHC in the Working Group 3 Yellow Report [3]. Thanks to this work a harmonised choice of the simulation and of the uncertainties treatment is adopted.

\section{Supersymmetry searches}

An extensive program is planned in the search for signatures predicted by theories of supersymmetry (SUSY). The larger dataset will naturally increase the analyses sensitivity and allows for stricter selection criteria in order to probe uncovered phase space corners. In most of these scenarios HL-LHC will increase the Run-2 mass reach by $20-50 \%$.

Searches for direct production of light stau pairs at HL-LHC are motivated by both experimental and theoretical considerations, and in some scenarios this kind of production can dominate with respect to the production of squarks and gluinos at LHC. However, due to the very small crosssection of the process, the exclusion contour at 95\% CL with the full Run-2 dataset extends only up to $m_{\tau}=400 \mathrm{GeV}$ for a massless lightest neutralino in the scenario of direct production of stau pairs, with each stau decaying into the lightest neutralino and one tau lepton [4]. Figure 1a shows how the dataset of $3 \mathrm{ab}^{-1}$ increases the expected sensitivity by almost a factor of 2 than the present results $[5,6]$.

Another important set of signatures which can be accessible at HL-LHC arises from compressed scenarios. In the context of Higgsino-like lightest supersymmetric particles, if the lightest chargino $\left(\widetilde{\chi}_{1}^{ \pm}\right)$and second lightest neutralino $\left(\widetilde{\chi}_{2}^{0}\right)$ are close in mass, topologies with soft objects in the final states coming from the decay of an off-shell $Z$ or $W$ boson $\left(\widetilde{\chi}_{2}^{0} \rightarrow Z^{*} \widetilde{\chi}_{1}^{0}, \widetilde{\chi}_{1}^{ \pm} \rightarrow W^{*} \widetilde{\chi}_{1}^{0}\right)$, 
can be detected by requiring a recoiling initial-state-radiated (ISR) jet. Challenging lepton identification and trigger upgrades will be needed to maintain the low lepton thresholds and tag this kind of event. Even in this case the HL-LHC projections will largely improve the current results based on $140 \mathrm{fb}^{-1}$ collected in Run-2 [7], extending the $m_{\widetilde{\chi}_{2}^{0}}$ reach by more than a factor of 2 (Figure $1 b)[8,9]$.

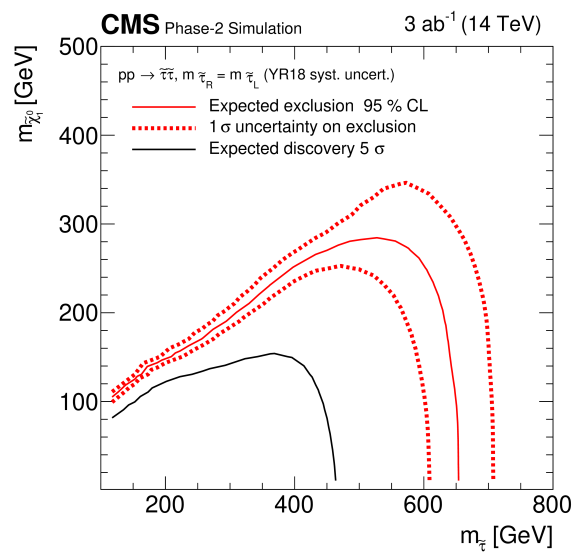

(a)

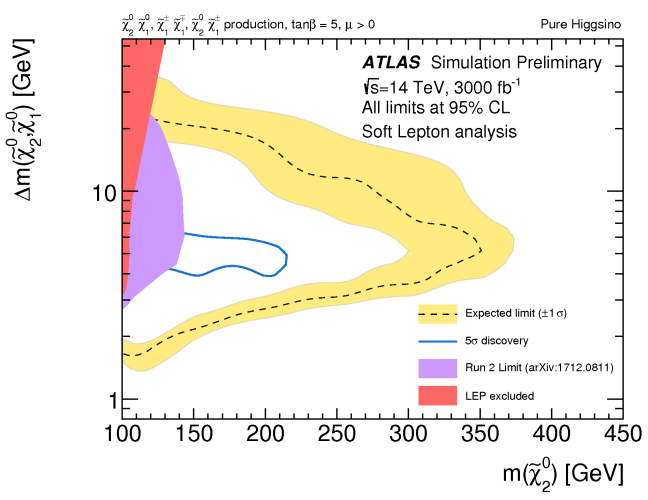

(b)

Figure 1: (a): expected upper limits at 95\% CL and the $5 \sigma$ discovery potential contour for the combination of the results of the $\tau_{h} \tau_{h}$ and $\ell \tau_{h}$ channels [6]. (b): expected exclusion limit (dashed line) in the $\Delta m\left(\widetilde{\chi}_{1}^{0}, \widetilde{\chi}_{2}^{0}\right), m_{\widetilde{\chi}_{2}^{0}}$ mass plane at $95 \%$ CL at HL-LHC in the context of a pure Higgsino LSP. The blue curve corresponds to the $5 \sigma$ discovery potential. The purple contour is the observed exclusion limit from the Run-2 analysis based on $36 \mathrm{fb}^{-1}$ [10]. The limits coming from the LEP results are shown in red [8].

\section{Dark Matter searches}

The search for Dark Matter (DM) candidates is an important part of the HL-LHC program. The key channel characterised by events with an energetic jet and missing transverse momentum $\left(E_{\mathrm{T}}^{\mathrm{miss}}\right)$ in the final state is fundamental to probe a vast range of BSM theories which predict particles invisible to the detectors. Since the main challenge in this channel is the treatment and constraining of the systematic uncertainties, the projections in different experimental and theoretical systematic uncertainty scenarios are shown in Figure 2a [11]. The results are interpreted in the simplified model in which two DM particles $(\chi)$ are produced through an axial-vector mediator $\left(Z_{A}\right)$ in the $s$-channel with couplings between mediator and quarks $\left(g_{q}\right)$ and mediator and DM particles $\left(g_{\chi}\right)$ set to $g_{q}=1$ and $g_{\chi}=0.25$. The prediction is based on the analysis performed with $36 \mathrm{fb}^{-1}$ at $13 \mathrm{TeV}$ [12]. An improvement of the systematic uncertainties related to the theoretical modelling of the signal and background processes (e.g. PDF uncertainties which will be reduced with a larger dataset) is found to give the leading contribution to sensitivity. By reducing the systematic uncertainties by a factor of 4 , the exclusion contours in the $\left(m_{\chi}, m_{Z_{A}}\right)$ mass plane at $95 \%$ CL can be extended up to $2.9 \mathrm{TeV}$ for DM particle masses of $1 \mathrm{GeV}$ (extending by more than $1 \mathrm{TeV}$ the current Run-2 results). 
More refined theories which cannot be probed by the $E_{\mathrm{T}}^{\text {miss }}+$ jet channel (such as the 2HDM+a model [13]) can be investigated by other $E_{\mathrm{T}}^{\text {miss }}+X$ channels, where $X$ represents $H, Z, \gamma$ or heavy flavour quarks [14-16]. In Figure $2 b$, the $E_{\mathrm{T}}^{\text {miss }}+Z$ channel sensitivity at $3 \mathrm{ab}^{-1}$ is shown for the $2 \mathrm{HDM}+\mathrm{a}$ model in the $\left(m_{H / A}, m_{a}\right)$ plane, in which $a$ represents the lightest pseudo-scalar higgs, whereas $H$ and $A$ the more massive bosons. Three different systematic scenarios are also shown for this case: by keeping the same uncertainties as in the current Run-2 results, by adopting the systematic uncertainty scheme used in the Yellow Report, and by neglecting them completely. The expected limits on $m_{a}$ at $95 \%$ CL increase by $50-100 \%$ relative to the limits of the current Run-2 search in the same channel.

Finally, the $\mathrm{VBF}+E_{\mathrm{T}}^{\text {miss }}$ channel topology, with events containing a pair of jets with high invariant mass, large pseudo-rapidity separation and small separation in azimuthal angle, is a golden channel for constraining the branching ratio of the higgs boson decay in invisible particles $(\mathscr{B}(h \rightarrow$ inv $))$. A large increase in sensitivity is expected by the tracker extension, however pile-up rejection is particularly challenging at HL-LHC and it will be a key point to reach a sensitivity to $\mathscr{B}(h \rightarrow$ inv $) \sim 5-10 \%[15,17]$.

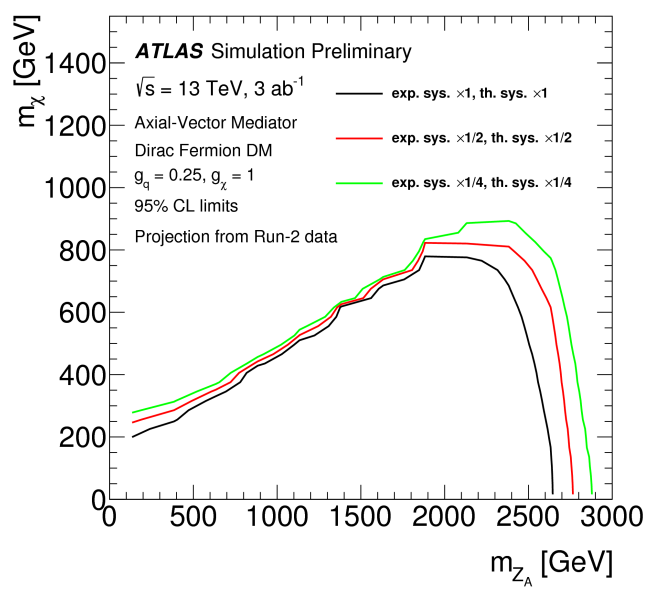

(a)

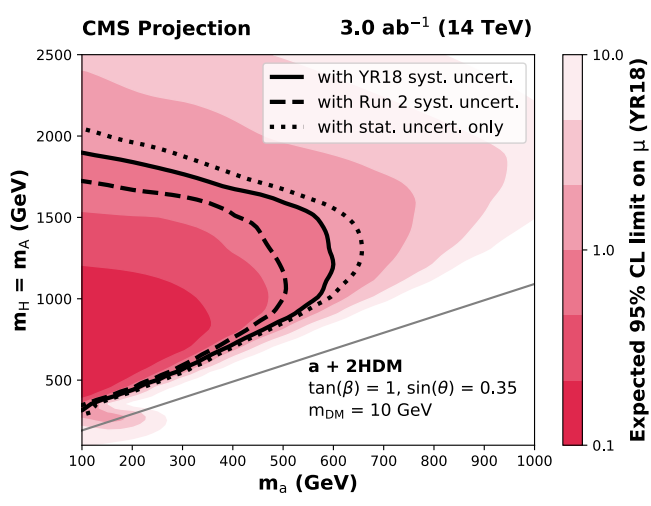

(b)

Figure 2: (a): expected 95\% CL excluded regions in the $E_{\mathrm{T}}^{\text {miss }}+$ jet on the $\left(m_{\chi}, m_{Z_{A}}\right)$ mass plane for the axial-vector simplified model with couplings $g_{q}=1$ and $g_{\chi}=0.25$. The three contours shown corresponds to three different systematic uncertainty scenarios: same used in [12] (black), same but reduced by a factor of 2 (red) and 4 (green) [11]. (b): expected 95\% CL exclusion limits on the signal strength in the $2 \mathrm{HDM}+\mathrm{a}$ scenario as a function of the mass of the main DM mediator $a$ and the masses of the $H$ and $A$ bosons. The results are shown for three systematic uncertainty scenarios [16].

\section{Resonance searches}

An extensive range of BSM models predict heavy exotic resonances and the search for them is one of the challenging objectives of the ATLAS and CMS experiments at HL-LHC. The dataset 
and collision energy will enhance the high mass reach which is currently limited by the statistical uncertainty. For example, in the $Z^{\prime}$ and $W^{\prime}$ Sequential Standard Model (SSM) scenarios, in which the heavy vector bosons can decay to leptons, the mass reach will be extended up to 6.5$7.9 \mathrm{TeV}[18,19]$, increasing by about $1.4-2.1 \mathrm{TeV}$ relative to the current full Run-2 results [20,21]. Figure 3a shows the transverse mass distribution for events satisfying all selection criteria of the search for $W_{\text {SSM }}^{\prime} \rightarrow e v$. In order to satisfy these projections, excellent performance of high- $p_{\mathrm{T}}$ objects are needed. In hadronic decays of exotic resonances, top final states dominate, and the new tracking and TDAQ capabilities should guarantee good $b$-tagging efficiency and low mis-ID probability in high pileup regimes. In Figure 3b, the projection of the 95\% CL expected upper limits for a Randall-Sundrum Kaluza-Klein gluon (RSG) decaying to $t \bar{t}$ shows a sensitivity to masses up to $6.6 \mathrm{TeV}$ [22], which extends the current limit of $4.5 \mathrm{TeV}$ based on Run-2 data [23].

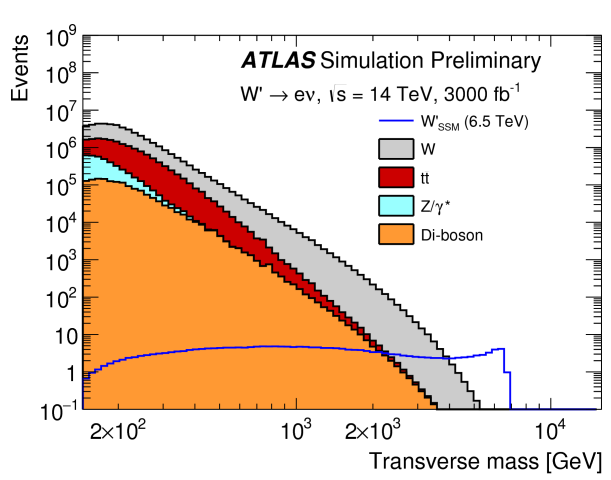

(a)

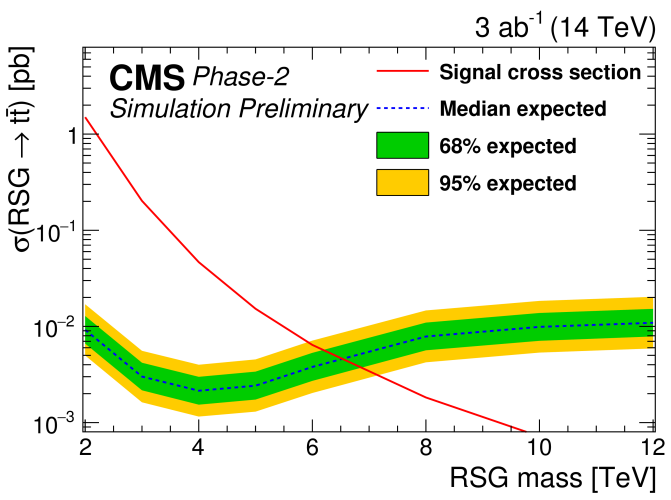

(b)

Figure 3: (a): transverse mass distribution in the signal region of the search for $W_{\mathrm{SSM}}^{\prime} \rightarrow e v$ [19]. (b): expected $95 \%$ CL exclusion upper limit of the cross-section of a RSG resonance decaying to $t \bar{t}$ for the combined single-lepton and fully hadronic final states [22].

\section{Long-lived particle searches}

Detector upgrades at HL-LHC will open new prospects for long-lived particle (LLP) searches. Generally, the searches are statistically limited and often background-free, hence they will naturally gain significantly in sensitivity with the foreseen dataset. In the ATLAS experiment the installation of the new inner tracker (ITk) $[24,25]$ will increase the acceptances and efficiencies thanks to the improved geometry, the larger silicon volume and the lower material budget. Figure 4 compares the layout of the Run-2 ATLAS inner tracker and ITk detector of the HL-LHC era [26].

In Figure 5a the comparison of displaced vertex acceptance for a R-hadron decay is shown in the Run-2 and HL-LHC conditions. The extended reach in terms of lifetime is due to the increased acceptance. The full analysis, which looks at the signature of a displaced vertex within the tracker together with moderate $E_{\mathrm{T}}^{\text {miss }}$, extends the reach for lifetimes of $10 \mathrm{~ns}$ up to gluino masses of about 3.4 TeV, more than $1 \mathrm{TeV}$ better than the Run-2 results with $33 \mathrm{fb}^{-1}$ of data (Figure 5b) [27].

The installation of new detectors can also permit new specialised techniques relevant for LLP searches. An example is the CMS precision MIP timing detector (MTD) which allows a time to 


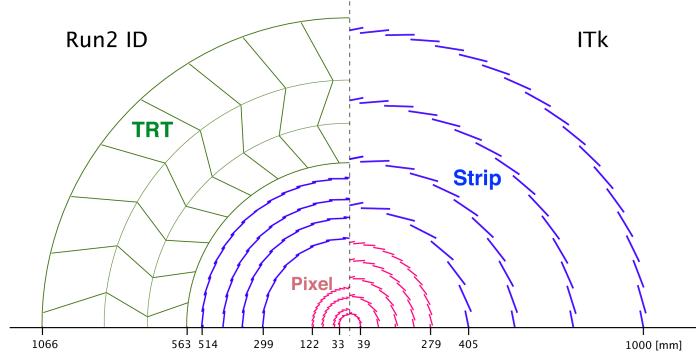

Figure 4: Layout comparison of the ATLAS Run-2 inner trackers and the ITk detector [26].

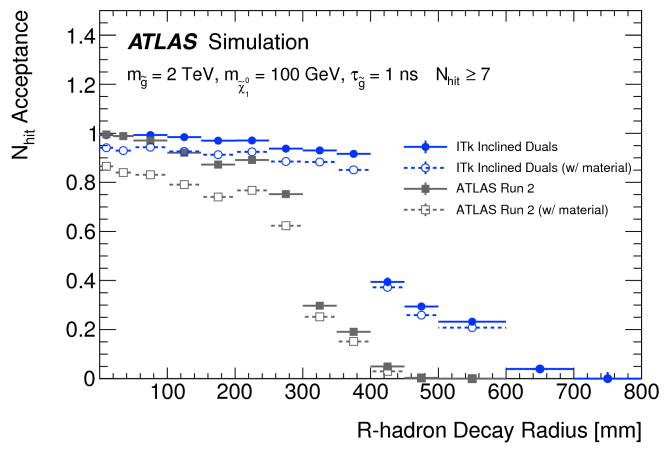

(a)

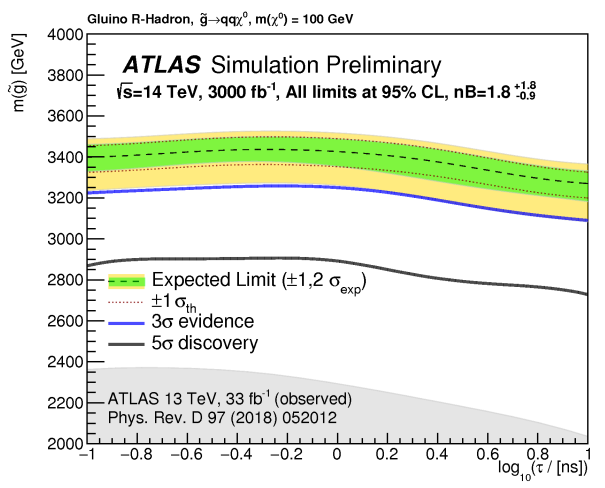

(b)

Figure 5: (a): probability that a charged particle, with $p_{\mathrm{T}}>1 \mathrm{GeV}$ produced in the decay of a $2 \mathrm{TeV}$ R-hadron with lifetime of $1 \mathrm{~ns}$, passes through at least seven silicon layers, as a function of the decay radius of the R-hadron, for both the Run 2 and ITk detectors. The probability is also shown without the simulated effect of material interactions [26]. (b): HL-LHC projection of the excluded upper limit at $95 \% \mathrm{CL}$ on the mass of a gluino R-hadron as a function of the gluino lifetime. Discovery reaches at $3 \sigma$ and $5 \sigma$ are shown alongside the observed ATLAS exclusion limits for a Run-2 dataset of $33 \mathrm{fb}^{-1}$ [26].

be assigned to each reconstructed vertex and to measure the time of flight (TOF) of LLPs between primary and secondary vertices [28]. Furthermore precision timing information helps to reject spurious secondary vertices and pile-up tracks. In the gauge-mediated SUSY breaking scenario, the lightest neutralino $\left(\widetilde{\chi}_{1}^{0}\right)$ is the next-to-lightest supersymmetric particle and can be long-lived. When it decays to a photon and the LSP gravitino $(\widetilde{G})$, the time of arrival of the photon at the detector can be used to discriminate signal from background. Measurements of an ISR jet with the MTD detector can reduce the primary vertex time resolution to $\sigma_{t}=30 \mathrm{ps}$, an improvement of a factor of 10 with respect to using the CMS electromagnetic calorimeter information alone. Figure 6 shows the 95\% CL exclusion contours, based on different timing resolutions, in the $\Lambda$ scale parameter (related to gravitino mass) versus the neutralino lifetime. The sensitivity improvement is particularly relevant at shorter lifetimes.

Finally upgrades foreseen for muon spectrometers in ATLAS and CMS can also substantially increase the detection efficiencies for LLP signatures. In CMS, the new RPC link boards will 


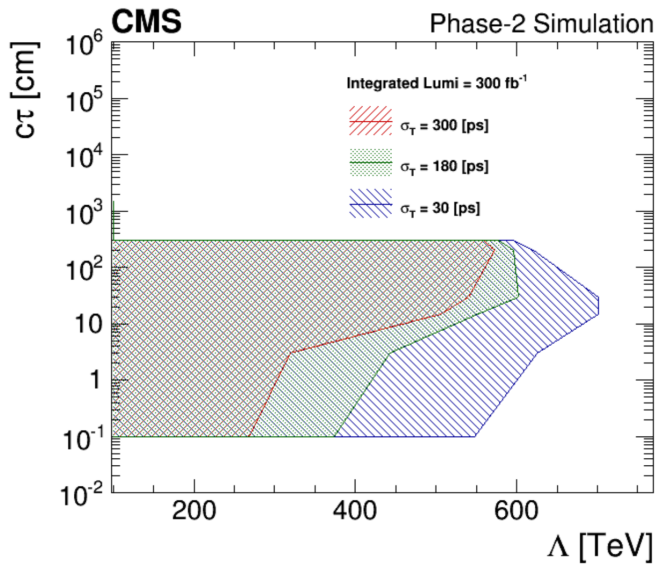

Figure 6: $95 \% \mathrm{CL}$ exclusion contours for $\widetilde{\chi}_{1}^{0} \rightarrow \widetilde{G} \gamma$ signatures predicted by the GMSB theory expressed in terms of neutralino lifetimes and $\Lambda$ scale, assuming different timing resolution which can be obtained by the current and upgradated CMS electromagnetic calorimeter and by the MTD detector. The projections are based on an integrated luminosity of $300 \mathrm{fb}^{-1}$ [28].

improve the hit time resolution by a factor of 25 , making it possible to measure the TOF of heavy stable charged particles, which are similar to muons but much slower [29]. In ATLAS, the improved coverage due to an additional inner RPC layer will increase by about $10 \%$ the single muon trigger efficiency coming from a dark-photon, as can be observed in Figure 7a [30]. Decays of this kind of object produce displaced collimated jets of muons. Furthermore, dedicated algorithms to select multi-muons in single region of interest would then allow the Level-0 muon $p_{\mathrm{T}}$ threshold to be reduced from 20 to $10 \mathrm{GeV}$, increasing the signal efficiency by $\sim 10 \%$ for dark-photon lifetimes on the order of $50 \mathrm{~mm}$, as can be observed in Figure $7 \mathrm{~b}$.

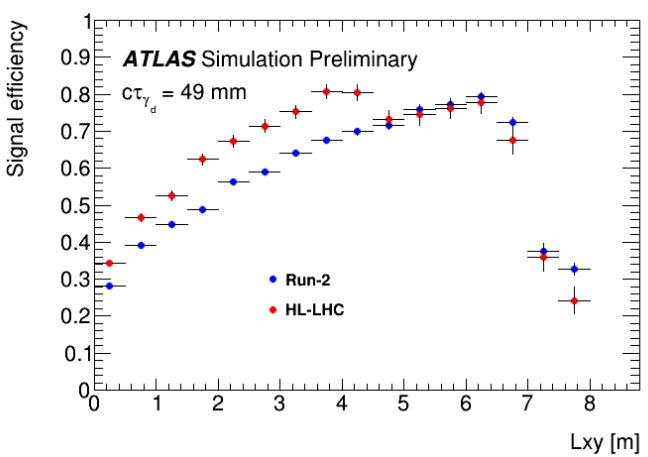

(a)

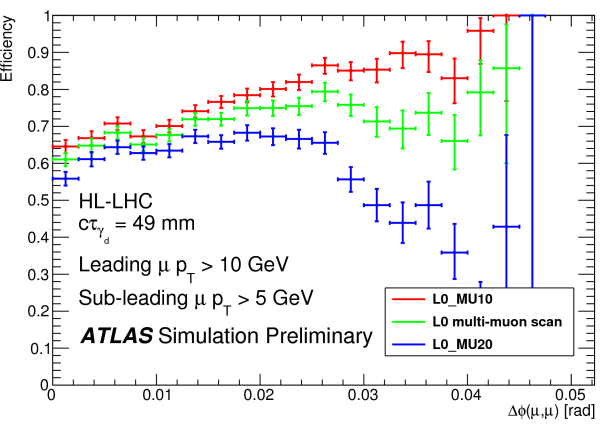

(b)

Figure 7: (a): muon trigger efficiency for $p_{\mathrm{T}}=20 \mathrm{GeV}$ Run-2 (blue) and the HL-LHC (red) low level muon trigger as a function of the truth transverse decay position of the dark photon [30]. (b): efficiency for different trigger selections as a function of the opening angle of two muons of the $\gamma_{d}$ decay. Single muon triggers with 10 and $20 \mathrm{GeV} p_{\mathrm{T}}$-threshold are shown in red and blue, respectively. The L0 multi-muon scan trigger is shown in green [30]. 


\section{Conclusions}

HL-LHC provides a unique opportunity to probe new physics scenarios which could explain some poorly understood phenomena existing in nature. A large gain in most of the BSM searches is expected from the higher $p p$ collision energy and the expected amount of data delivered, which lead in turn to reduced theoretical and experimental uncertainties (e.g. coming from the PDFs calculation accuracy or from the object resolutions at extreme energy regimes). Furthermore, the upgraded ATLAS and CMS detectors will open new opportunities to develop new techniques and strategies which help to tag unprobed or unconventional signatures. The prospects of several new physics scenarios have been collected in the Yellow Report [3], but much work is required in order to fully exploit the vast physics potential of the HL-LHC.

\section{References}

[1] ATLAS Collaboration, JINST 3, S08003 (2008).

[2] CMS Collaboration, JINST 3, S08004 (2008).

[3] X. Cid Vidal et al. [Working Group 3], arXiv:1812.07831 [hep-ph].

[4] ATLAS Collaboration, ATLAS-CONF-2019-018.

[5] ATLAS Collaboration, ATL-PHYS-PUB-2018-048.

[6] CMS Collaboration, CMS-PAS-FTR-18-010.

[7] ATLAS Collaboration, ATLAS-CONF-2019-014.

[8] ATLAS Collaboration, ATL-PHYS-PUB-2018-031.

[9] CMS Collaboration, CMS-PAS-FTR-18-001.

[10] ATLAS Collaboration, Phys. Rev. D 97, no. 5, 052010 (2018).

[11] ATLAS Collaboration, ATL-PHYS-PUB-2018-043.

[12] ATLAS Collaboration, JHEP 1801, 126 (2018).

[13] M. Bauer, U. Haisch and F. Kahlhoefer, JHEP 1705 (2017) 138.

[14] ATLAS Collaboration, ATL-PHYS-PUB-2018-024.

[15] ATLAS Collaboration, ATL-PHYS-PUB-2018-038.

[16] CMS Collaboration, CMS-PAS-FTR-18-007.

[17] CMS Collaboration, CMS-PAS-FTR-18-016.

[18] ATLAS Collaboration, ATL-PHYS-PUB-2018-022.

[19] ATLAS Collaboration, ATL-PHYS-PUB-2018-044.

[20] ATLAS Collaboration, Phys. Lett. B 796, 68 (2019)

[21] ATLAS Collaboration, arXiv:1906.05609 [hep-ex].

[22] CMS Collaboration, CMS-PAS-FTR-18-009.

[23] CMS Collaboration, JHEP 1904, 031 (2019). 
[24] ATLAS Collaboration, CERN-LHCC-2017-021.

[25] ATLAS Collaboration, CERN-LHCC-2017-021.

[26] ATLAS Collaboration, ATL-PHYS-PUB-2018-033.

[27] ATLAS Collaboration, Phys. Rev. D 97, no. 5, 052012 (2018).

[28] CMS Collaboration, CERN-LHCC-2017-027.

[29] CMS Collaboration, CERN-LHCC-2017-012.

[30] ATLAS Collaboration, ATL-PHYS-PUB-2019-002. 